\title{
The matrikine acetyl-proline-glycine-proline and clinical features of COPD: findings from SPIROMICS
}

J. Michael Wells ${ }^{1,2,3^{*}}$, Dongqi Xing ${ }^{1,2}$, Liliana Viera ${ }^{1,2}$, Robert M. Burkes ${ }^{4,5}$, Yixin Wu ${ }^{1,2}$, Surya P. Bhatt ${ }^{1,2}$, Mark T. Dransfield ${ }^{1,2,3}$, David J. Couper ${ }^{6}$, Wanda O'Neal ${ }^{5}$, Eric A. Hoffman ${ }^{7}$, Amit Gaggar ${ }^{1,2,3}$, Igor Barjaktarevic ${ }^{8}$, Jeffrey L. Curtis ${ }^{9,10}$, Wassim W. Labaki ${ }^{9}$, Mei Lan K. Han', Christine M. Freeman ${ }^{9,10}$, Nirupama Putcha ${ }^{11}$,

Thomas Schlange ${ }^{12}$, J. Edwin Blalock ${ }^{1,2}$ and for the SPIROMICS Investigators,

\begin{abstract}
Background: Pulmonary and systemic inflammation are central features of chronic obstructive pulmonary disease (COPD). Previous studies have demonstrated relationships between biologically active extracellular matrix components, or matrikines, and COPD pathogenesis. We studied the relationships between the matrikine acetylproline-glycine-proline (ACPGP) in sputum and plasma and clinical features of COPD.

Methods: Sputum and plasma samples were obtained from COPD participants in the SPIROMICS cohort at enrollment. AcPGP was isolated using solid phase extraction and measured by mass spectrometry. Demographics, spirometry, quality of life questionnaires, and quantitative computed tomography (CT) imaging with parametric response mapping (PRM) were obtained at baseline. Severe COPD exacerbations were recorded at 1-year of prospective follow-up. We used linear and logistic regression models to measure associations between AcPGP and features of COPD, and Kaplan-Meier analyses to measure time-to-first severe exacerbation.

Results: The 182 COPD participants in the analysis were $66 \pm 8$ years old, $62 \%$ male, $84 \%$ White race, and 39\% were current smokers. ACPGP concentrations were $0.61 \pm 1.89 \mathrm{ng} / \mathrm{mL}$ (mean $\pm \mathrm{SD}$ ) in sputum and $0.60 \pm 1.13 \mathrm{ng} / \mathrm{mL}$ in plasma. In adjusted linear regression models, sputum AcPGP was associated with $F E V_{1} / F V C$, spirometric GOLD stage, PRM-small airways disease, and PRM-emphysema. Sputum ACPGP also correlated with severe AECOPD, and elevated sputum AcPGP was associated with shorter time-to-first severe COPD exacerbation. In contrast, plasma AcPGP was not associated with symptoms, pulmonary function, or severe exacerbation risk.
\end{abstract}

Conclusions: In COPD, sputum but not plasma AcPGP concentrations are associated with the severity of airflow limitation, small airways disease, emphysema, and risk for severe AECOPD at 1-year of follow-up.

Trial registration: ClinicalTrials.gov: NCT01969344 (SPIROMICS).

Keywords: COPD, Acetyl proline-glycine-proline (ACPGP), Sputum, Matrikine, Inflammation, Biomarker

\footnotetext{
* Correspondence: jmwells@uabmc.edu

${ }^{1}$ Division of Pulmonary, Allergy, and Critical Care Medicine, University of

Alabama at Birmingham, Birmingham, AL, USA

${ }^{2}$ UAB Lung Health Center, Birmingham, AL, USA

Full list of author information is available at the end of the article
}

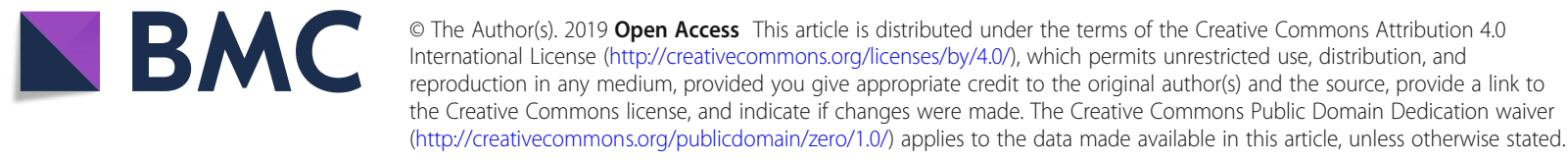




\section{Introduction}

Chronic obstructive pulmonary disease (COPD) is a progressive inflammatory disease affecting the airways and lung parenchyma, often as the result of chronic cigarette smoking. COPD is classified based on symptoms, lung function impairment, and risk for acute exacerbations of COPD (AECOPD) [1]. There remains a need to identify biomarkers that reflect the underlying molecular and cellular processes responsible for development of lung destruction (i.e. emphysema and airway remodeling) and clinical features including rapid lung function decline, chronic bronchitis, and exacerbation risk [2].

We have previously identified a matrikine (a biologically active extracellular matrix peptide), acetylated proline-glycine-proline (AcPGP), as a pathogenic regulator of cigarette-smoke-mediated emphysema development in animal models and human smokers [3, 4]. In this pathway, AcPGP is generated by the stepwise proteolytic cleavage of collagen by matrix metalloproteases (MMPs) and prolyl endopeptidase (PE) and avoids degradation by direct effects of cigarette-smoke on leukotriene A4 hydrolase [3, 5-7]. AcPGP, as a matrikine, stimulates neutrophil recruitment to sites of inflammation in the lung and propagates a feed-forward cycle of inflammation. Although our previous work has clearly demonstrated the role of the AcPGP-pathway in disease pathogenesis [3, 4], evaluation of the relevance of AcPGP in longitudinal examination of clinical features of COPD including pulmonary function, CT features, symptoms, and exacerbations is required. Additionally, our previous work has primarily focused on measuring AcPGP in the sputum $[4,8]$, while little focus has been put on evaluating the relevance of plasma AcPGP in COPD.

We hypothesized that the matrikine AcPGP, as a marker of lung inflammation, will be associated with meaningful clinical features of COPD including lung function impairment, emphysema, symptoms, and risk for AECOPD. We tested this hypothesis using plasma and sputum samples from participants with COPD enrolled in the SubPopulations and InteRmediate Outcome Measures in COPD Study (SPIROMICS) cohort.

\section{Methods}

\section{Subjects}

The design of SPIROMICS (ClinicalTrials.gov NCT01 969344) has been described [9]. Briefly, SPIROMICS is a multi-center prospective observational study to identify unique biomarkers and phenotypes that can be used as intermediate outcomes to reliably predict clinical benefits in future clinical trials. SPIROMICS enrolled participants between November 2011 and January 2015. COPD was defined as a post-bronchodilator forced expiratory volume in 1 -s $\left(\mathrm{FEV}_{1}\right) /$ forced vital capacity $(\mathrm{FVC})<0.70$ [10]. Participants underwent baseline and in-person follow-up visit 12-months later. Clinical data reported here include results from the SPIROMICS Core5 dataset. For these studies, we report data from subjects with COPD, complete clinical information, and blood and sputum AcPGP measurements. This study was approved by the University of Alabama at Birmingham IRB (X110921005).

\section{Blood and sputum collection and processing}

Participants with a post-bronchodilator $\mathrm{FEV}_{1} \geq 35 \%$ predicted were eligible to undergo sputum induction using nebulized saline solutions administered via ultrasonic nebulizer as previously described [11] and as outlined in the Supplementary Methods. Briefly, the saline solutions were given in three 7-min intervals and sputum was immediately processed using a 1:4 (weight:volume) $0.1 \%$ sputolysin solution followed by an additional 1:4 (volume: volume) $1 \mathrm{mM}$ EDTA solution. Plasma was collected in tubes containing EDTA and was immediately processed and shipped to the Genomics and Informatics Center (GIC) at the University of North Carolina at Chapel Hill (UNC).

\section{AcPGP measurement}

Plasma was prepared by solid phase extraction using Phree Phospholipid Removal Columns (Phenomenex, Torrence, CA, USA). First, columns were washed with a methanol:acetonitrile $(60: 40)$ solution. Next, an internal standard peptide (IS) $\left({ }^{13 \mathrm{C}, 15 \mathrm{~N}} \mathrm{PGP} /{ }^{13 \mathrm{C}, 15 \mathrm{~N}} \mathrm{AcPGP}\right) \mathrm{mix}$ ture was added to the plasma sample; plasma and IS were then placed on the Phree column. Columns were centrifuged at $4{ }^{\circ} \mathrm{C}$ for $60 \mathrm{~min}$ at $1300 \mathrm{xG}$ followed by an additional methanol:acetonitrile wash, re-centrifugation, and collection. Afterwards, samples underwent evaporation using a Nitrogen evaporator. Dried plasma samples were then reconstituted using PBS. Sputum samples were prepared as follows: $10,000 \mathrm{kDa}$ molecular weight cutoff filters were prepared by washing with an ethanol: water $(65: 45)$ solution. Next, IS was added to the sputum sample; this sputum-IS mixture was then added to the washed filters and centrifuged at $4{ }^{\circ} \mathrm{C}$ for $30 \mathrm{~min}$ at 12 , 500 xG. Finally, samples were washed using $1 \mathrm{mM} \mathrm{HCl}$ followed by centrifugation and collection of supernatants. AcPGP was measured by tandem mass spectrometry (MS/MS) as previously described $[3,4,7,8,12]$.

\section{Pulmonary function}

Pulmonary function testing was performed according to the SPIROMICS protocol and ATS/ERS criteria $[9,10]$ and post-bronchodilator values were recorded using a KoKo spirometer (nSpire Health, Longmont, Co.). Participants were stratified according to Global Initiative for Chronic Obstructive Lung Disease (GOLD) stage [1]. 
Health status and respiratory symptoms

We assessed health status and quality of life using the COPD Assessment Test (CAT) [13] and the St. George's Respiratory Questionnaire (SGRQ) [14], and dyspnea using the Modified Medical Research Council Questionnaire (MMRC) [15]. Chronic bronchitis was defined by answers to chronic cough and phlegm questions on the SGRQ [16]. Six minute walk tests were performed following ATS guidelines [17].

\section{Severe exacerbations}

Given the impact of severe AECOPD on re-hospitalization and mortality [18], we evaluated associations between these events and sputum AcPGP. Prior severe AECOPD was defined as self-reported hospitalization for AECOPD that occurred within the 12-months preceding the baseline study visit. Prospective severe AECOPD were recorded from the time of the baseline study visit through the first year of follow-up in SPIROMICS. Severe AECOPD were selfreported during quarterly phone calls and were defined as a worsening of respiratory symptoms lasting longer than $48 \mathrm{~h}$ that warranted an emergency department visit or hospitalization for treatment of acute respiratory disease [19]. During the phone calls, participants (or their representatives) were asked "Since your last [visit or phone contact on [date], have you had a flare-up of chest trouble?"; positive responses were followed up with questions ascertaining the number of events, treatments for each episode (antibiotics, steroids, both, unsure, or can't remember); participants were asked "Were you evaluated in an Emergency Department?" followed by questions on treatments; participants were then asked "Were you admitted to the hospital?" and further information was collected about dates and location of the medical facility as well as treatments given during the hospitalization.

\section{Radiologic measurements}

The methods for quantitative computed tomography (CT) were published previously [20]. Briefly, inspiratory and expiratory lung CT scans were performed at the baseline SPIROMICS visit. Parametric Response Mapping (PRM) (Imbio, Minneapolis, MN) was used to calculate amounts of emphysema (PRM ${ }^{\text {emph}}$ ) and functional small airways disease $\left(\mathrm{PRM}^{\mathrm{fSAD}}\right)$ as previously defined $[21,22]$. This technique was recently demonstrated to correlate significantly with histologically-confirmed small airways disease in lung specimens from patients with advanced COPD [23].

\section{Statistical analyses}

Due to the study design, there were only 2 participants with GOLD spirometry stage 4 who underwent sputum induction; therefore, spirometric GOLD stages 3 and 4 were combined into a single group defined as severe airflow obstruction. Sputum and plasma AcPGP was divided into quartiles; elevated sputum or plasma AcPGP were defined as values above the median. Descriptive statistics, including means and standard deviations for continuous data, frequencies and percentages for categorical data, were calculated for all study variables of interest. Bivariate analyses were conducted by using the unpaired t-test for normally distributed continuous variables, Wilcoxon rank-sum test for continuous variables that were not normally distributed, or the chi-square test for categorical variables. Spearman's rho was used to measure correlations between sputum AcPGP and lung function measured by pulmonary function testing. Analysis of variance (ANOVA) was used to compare sputum AcPGP values across spirometric GOLD stages. Associations between AcPGP and pulmonary function tests were explored using linear regression models adjusted for age, sex, and current smoking status. Additional linear regression models were further adjusted for FEV1 percent predicted to measure associations between AcPGP and quality of life assessments and quantitative CT measurements. To identify associations between sputum or plasma AcPGP and any severe AECOPD at 1-year of follow-up, we used logistic regression models, adjusted for age, sex, $\mathrm{FEV}_{1}$ percent predicted, prior severe AECOPD (within one year before enrollment), and current smoking status. Kaplan-Meier survival analysis with log-rank test was used to identify time-to-first severe AECOPD based on the presence or absence of elevated sputum AcPGP. All statistical tests were two-sided and were performed using a significance level of $P<0.05$. Statistical analyses were conducted using SPSS software (Version 23, IBM Corporation).

\section{Results}

\section{Characteristics of the participants}

We measured sputum and plasma AcPGP at the baseline SPIROMICS visit in 271 participants, including 182 subjects with COPD. The CONSORT diagram is shown in Additional file 1 Figure S1. We limited this analysis to participants with COPD; information on the excluded participants without COPD is shown in Additional file 1. Participants with COPD were $66 \pm 8$ years old (mean $\pm \mathrm{SD}$ ), $62 \%$ male, $84 \%$ white race, had a post-bronchodilator $\mathrm{FEV}_{1}$ percent predicted $68 \pm 21$, and $39 \%$ were current smokers (Table 1). Among COPD subjects, concentrations of AcPGP were $0.60 \pm$ $1.13 \mathrm{ng} / \mathrm{mL}$ in plasma and $0.61 \pm 1.89 \mathrm{ng} / \mathrm{mL}$ in sputum. Subjects were generally symptomatic, with CAT scores of $14 \pm 8$, SGRQ scores of $33 \pm 19$, and more than $50 \%$ had chronic bronchitis. Ten percent $(n=18)$ had a self-reported previous severe AECOPD in the previous 12-months before the baseline visit. 
Table 1 Baseline Characteristics

\begin{tabular}{ll}
\hline & Cohort $(n=182)$ \\
\hline Age, years & $66 \pm 8$ \\
Male sex & $112(62 \%)$ \\
White race & $153(84 \%)$ \\
FEV1, percent predicted & $68 \pm 21$ \\
FVC, percent predicted & $94 \pm 19$ \\
FEV1/FVC & $0.54 \pm 0.11$ \\
GOLD Stage & \\
GOLD 1 & $48(26.4 \%)$ \\
GOLD 2 & $88(48.4 \%)$ \\
GOLD 3 & $44(24.2 \%)$ \\
GOLD 4 & $2(1.0 \%)$ \\
Current Smoker & $70(39 \%)$ \\
Pack-year history & $51 \pm 21$ \\
CAT & $14 \pm 8$ \\
SGRQ score, total & $33 \pm 19$ \\
CB-SGRQ & $101 / 173(56 \%)$ \\
MMRC dyspnea score & $1[0-1]$ \\
6-min walk distance, m & $412 \pm 101$ \\
PRM-emph (\%) & $7.3 \pm 10.4$ \\
PRM-fSAD (\%) & $23.4 \pm 11.7$ \\
Severe AECOPD in the previous year & $18 / 175(10 \%)$ \\
Plasma AcPGP ng/ml & $0.60 \pm 1.13$ \\
Sputum AcPGP ng/ml & $0.61 \pm 1.89$ \\
\hline Data exprss as mean &
\end{tabular}

Data expressed as mean \pm S.D.; median $[I \mathrm{QR}] ;$ or $\mathrm{n}(\%)$

\section{Sputum ACPGP and COPD symptoms and severity}

Participants with severe airflow obstruction (GOLD spirometry stage 3-4) had higher mean sputum AcPGP concentrations compared to individuals with mild-tomoderate obstruction (GOLD 1-2; $0.98 \pm 1.02$ vs $0.52 \pm$ $2.11 \mathrm{ng} / \mathrm{ml} ; P=0.05)$. There were no associations between sputum AcPGP and GOLD 2017 (ABCD) stages. There were significant correlations between sputum AcPGP quartiles and spirometric GOLD stage (Spearman's Rho $=0.20$;
$P=0.007), \mathrm{FEV}_{1}$ percent predicted (Spearman's rho $=-$ $0.14, P=0.041$ ), and FEF25-75\% predicted (Spearman's rho $=-0.18, P=0.016)$, but not with FVC percent predicted $(P=0.49)$ or FEV1/FVC $(P=0.069)$. The distribution of log-transformed AcPGP in sputum and plasma across spirometric GOLD stages is shown in Fig. 1. Sputum AcPGP was not correlated to mean sputum neutrophil count $(P=0.14)$.

In multiple linear regression models adjusted for age, sex, and current smoking status, sputum AcPGP remained independently associated with $\mathrm{FEV}_{1} / \mathrm{FVC}$, FEF25-75, and spirometric GOLD stage (Table 2). Sputum AcPGP was associated with PRM $^{\text {emph }}$ (Beta 1.72, SE $0.70, P=0.015$ ) and $\mathrm{PRM}^{\mathrm{fSAD}}$ (Beta 1.67, SE 0.81, $P=$ $0.040)$ in similarly adjusted linear regression models. In separate models adjusting for the above covariates plus FEV1\%, associations between sputum AcPGP and PRMemphysema remained statistically significant.

Sputum AcPGP was not associated with health status as measured by CAT, SGRQ (data not shown), or with dyspnea as measured by MMRC scores (data not shown). Nor was there a difference in mean sputum AcPGP among individuals with chronic bronchitis compared to those without chronic bronchitic symptoms $(0.53 \pm 1.18$ versus $0.79 \pm 2.78 \mathrm{ng} / \mathrm{mL}, P=0.44$ by Chi square testing).

\section{Sputum AcPGP and severe exacerbations}

After 1-year of follow-up, 10\% $(n=18 / 173)$ individuals had at least one severe AECOPD (median 0 events/year, range 0-3). Although sputum AcPGP was not statistically significantly different in participants that had a selfreported prior severe AECOPD $(P=0.21)$, sputum AcPGP was significantly associated with a severe AECOPD at 1year of follow-up $(P=0.019)$ (Table 3). Likewise, 83\% (15/ 18) of participants that had a severe AECOPD during follow-up had sputum AcPGP above the median values compared to $45 \%(69 / 155)$ who did not have a severe AECOPD $(P=0.002)$. In multivariable logistic regression
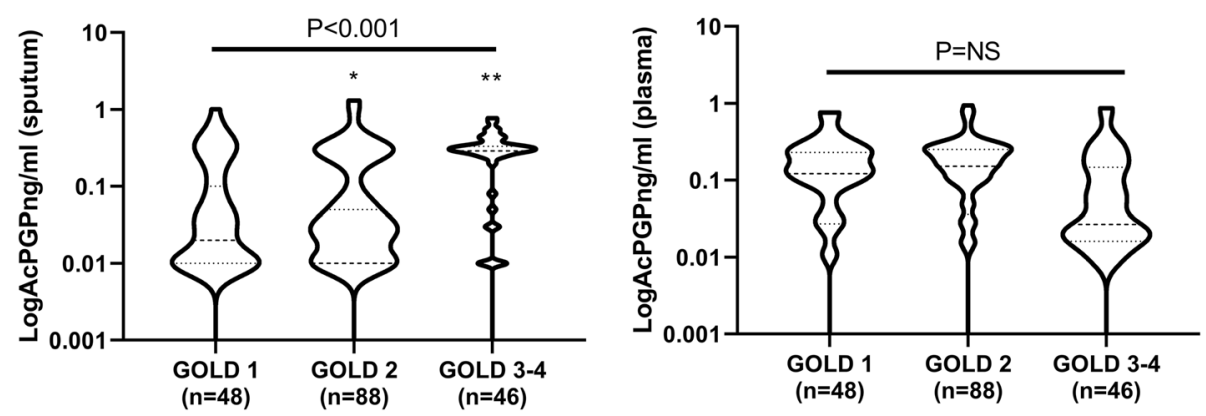

Fig. 1 Associations between ACPGP and COPD severity. Log-transformed ACPGP in A) sputum was significantly higher in GOLD 3/4 COPD compared to GOLD 1 or 2 but there were no differences in plasma AcPGP across spirometric GOLD stages. 1-way ANOVA with Tukey's post-hoc testing was used for analyses; ${ }^{*} P=0.0019$ between GOLD 1 and GOLD 3/4; ${ }^{*} P<0.0001$ between GOLD 2 and GOLD 3/4 
Table 2 Associations between Sputum AcPGP and clinical features of COPD

\begin{tabular}{|c|c|c|c|c|c|c|}
\hline & Model & & & Mode & & \\
\hline & Beta & S.E. & $P$-value & Beta & S.E. & P-value \\
\hline FEV1, percent predicted & -2.58 & 1.38 & 0.063 & $\mathrm{n} / \mathrm{a}$ & $\mathrm{n} / \mathrm{a}$ & $\mathrm{n} / \mathrm{a}$ \\
\hline FEV1/FVC & -0.02 & 0.007 & 0.018 & $\mathrm{n} / \mathrm{a}$ & $\mathrm{n} / \mathrm{a}$ & $\mathrm{n} / \mathrm{a}$ \\
\hline FEF25-75, percent predicted & -2.40 & 1.20 & 0.046 & $\mathrm{n} / \mathrm{a}$ & $\mathrm{n} / \mathrm{a}$ & $\mathrm{n} / \mathrm{a}$ \\
\hline GOLD Stage & 0.14 & 0.05 & 0.004 & $\mathrm{n} / \mathrm{a}$ & $\mathrm{n} / \mathrm{a}$ & $\mathrm{n} / \mathrm{a}$ \\
\hline CAT & 0.93 & 0.52 & 0.073 & 0.55 & 0.49 & 0.27 \\
\hline PRM-emph & 1.72 & 0.70 & 0.015 & 1.26 & 0.63 & 0.040 \\
\hline PRM-fSAD & 1.67 & 0.81 & 0.040 & 1.08 & 0.70 & 0.127 \\
\hline
\end{tabular}

Model $1=$ Linear regression models included sputum AcPGP, age, sex, current smoking status. ${ }^{*}$ Model $2=$ Linear regression model was adjusted for FEV 1 percent predicted in addition to previously listed covariates (model 2 was not used for spirometric based outcome variables)

models adjusted for age, $\mathrm{FEV}_{1} \%$ predicted, prior severe AECOPD, and current smoking status (Table 4), for each higher quartile sputum AcPGP value there were $75 \%$ increased odds (OR 1.75; 95\% CI 1.04-2.97, $P=0.037$ ) of having a severe AECOPD in the next year; alternatively, if the baseline sputum AcPGP was above the median, there was a $486 \%$ increased odds of a subsequent severe AECOPD (OR 4.86; 95\% CI 1.28-18.4, P=0.02). Participants with elevated sputum AcPGP (above the median) also had shorter time-to-first severe AECOPD compared to individuals with non-elevated sputum AcPGP (195 days [95\% CI 139-252] versus 305 days [259-351], $P=0.030$ by log-rank test) (Fig. 2).

\section{Plasma AcPGP and clinical outcomes}

Sputum and plasma AcPGP concentrations in individual participants were not significantly correlated $(R=-0.07$; $P=0.82$ ). Unlike the associations observed between sputum AcPGP and features of COPD, plasma AcPGP was not associated with pulmonary function, GOLD stage, symptoms or quality of life, or risk for severe AECOPD (Fig. 1b; Tables 2-4).

\section{Discussion}

Results of this analysis of a sizeable prospective cohort provide the first direct evidence that matrikines present in the sputum of patients with COPD are associated with salient, measurable parameters that impact disease progression and morbidity. We clearly demonstrate that induced sputum AcPGP was related to more severe airflow limitation, emphysema, and small airways disease. Additionally, we observed that sputum AcPGP was associated with a significantly increased risk for severe AECOPD within the first year of follow-up when adjusted for known risk factors including previous severe exacerbation. Sputum AcPGP was not associated with respiratory symptoms or respiratory health status measurements. We did not observe any relationships between circulating AcPGP and COPD outcomes.

These findings provide clinically relevant evidence that AcPGP has the potential to serve as a biomarker for COPD, extending previous studies showing that AcPGP is present in sputum and bronchoalveolar lavage samples in individuals with COPD as compared to healthy controls or smokers without COPD [4, 8]. Identification of measurable endpoints including pulmonary function,

Table 3 Associations between AcPGP and COPD exacerbations

\begin{tabular}{lll}
\hline & No severe AECOPD $(n=155)$ & Severe AECOPD $(n=18)$ \\
\hline Sputum AcPGP & & \\
Q1 & $42(28 \%)$ & $2(11 \%)$ \\
Q2 & $41(27 \%)$ & $1(6 \%)$ \\
Q3 & $38(25 \%)$ & $7(39 \%)$ \\
Q4 & $31(20 \%)$ & $8(44 \%)$ \\
Sputum AcPGP above median & $69(45 \%)$ & $15(83 \%)$ \\
Plasma AcPGP & & \\
Q1 & $37(24 \%)$ & $5(28 \%)$ \\
Q2 & $38(25 \%)$ & $4(22 \%)$ \\
Q3 & $41(27 \%)$ & $4(22 \%)$ \\
Q4 & $39(25 \%)$ & $5(28 \%)$ \\
Plasma AcPGP above median & $73(47 \%)$ & $11(61 \%)$ \\
\hline
\end{tabular}

Data expressed as $\mathrm{n}$ (percent). Chi square testing was used for analyses 
Table 4 Associations between AcPGP and Severe COPD exacerbations

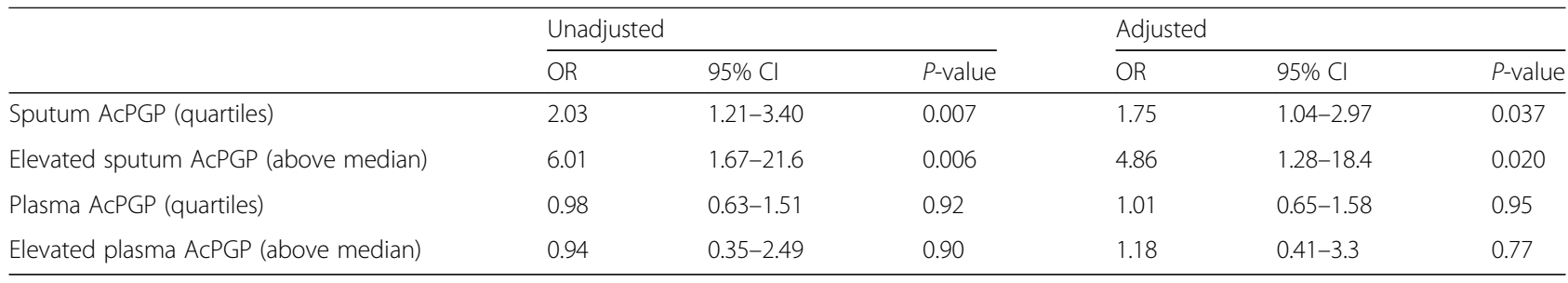

Logistic regression models were adjusted for age, sex, FEV1 percent predicted, prior severe AECOPD, and current smoking status

findings on CT imaging, and COPD exacerbations are vital to understanding the translational significance of biological pathways implicated in COPD pathogenesis. These observations provide links between mechanisms of neutrophil chemotaxis, pulmonary inflammation, and alveolar destruction and relevant translational endpoints. Our findings support sputum AcPGP as an indicator of COPD severity as well as a marker of increased odds for AECOPD development. Importantly, we have shown that sputum AcPGP is responsive to the use of daily azithromycin and roflumilast, two oral anti-inflammatory agents used for AECOPD risk reduction $[8,12]$. In both studies, treatment with either agent reduced sputum AcPGP as compared to individuals treated with placebo. Although in the MACRO study we previously observed temporal rise and fall in sputum AcPGP related to the time of an AECOPD, we did not find any correlation between AcPGP and pulmonary function or exacerbation risk, possibly due to the small sample size [12]. The current study addresses this gap in understanding the clinical relevance of this matrikine by directly linking it to lung structure/remodeling, pulmonary function, and prospective exacerbation risk.

The utility of sputum versus blood-based biomarkers in COPD is the subject of debate and ongoing investigation [2]. On one hand, blood-based assays are attractive to clinicians and investigators due to the wide availability of samples, ease in collection, standardized methodology, costs, and quality control issues as compared to induced sputum acquisition. However, it is not clear that blood-based biomarkers accurately reflect the active pathologic processes occurring in the lungs. One of the first major examples of this phenomenon in COPD was described by Singh and colleagues in the Evaluation of COPD Longitudinally to Identify Predictive Surrogate Endpoints (ECLIPSE) study. In that analysis, the investigators measured associations between sputum neutrophils as a potential biomarker for COPD among 488 participants [24]. They found sputum neutrophils were associated with pulmonary function and health status, but no association between sputum neutrophils and AECOPD or emphysema, suggesting a role for

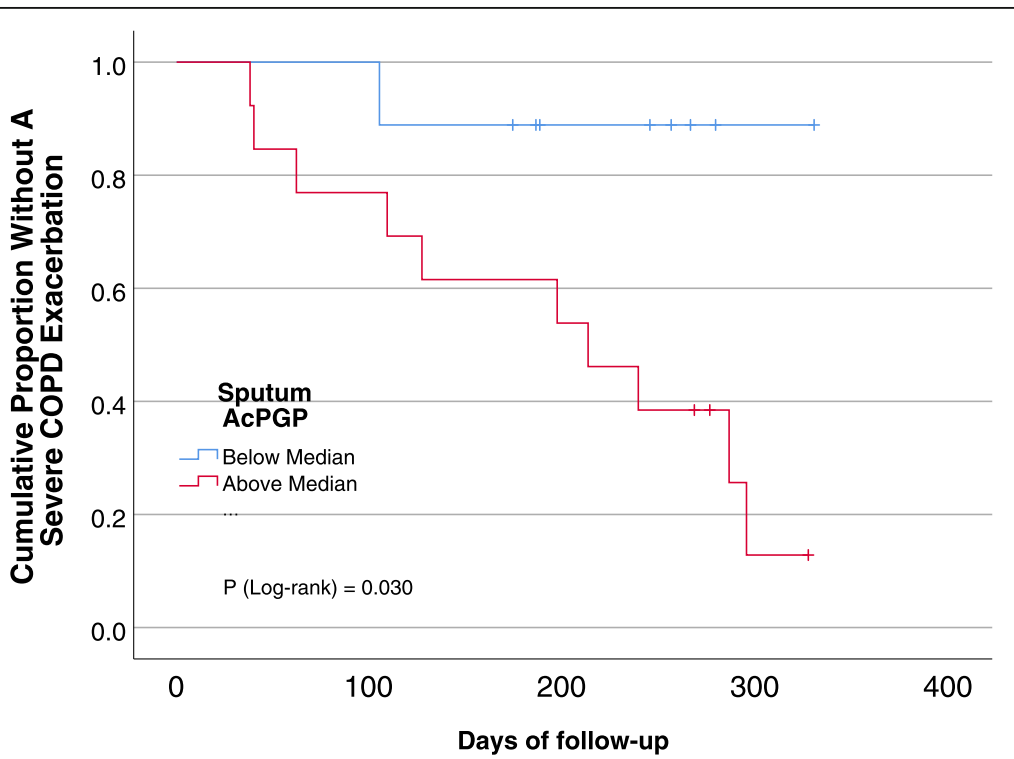

Fig. 2 Kaplan-Meier curve for severe AECOPD. COPD subjects with elevated sputum AcPGP had shorter time-to-first severe AECOPD compared to individuals with non-elevated sputum AcPGP (195 days [95\%Cl 139-252] versus 305 days [259-351], $P=0.030$ ) 
sputum based biomarkers in COPD. Likewise, Hastie and colleagues reported high degree of discordance between blood and sputum eosinophils in SPIROMICS, with sputum eosinophilic inflammation being a more robust biomarker for disease severity, exacerbation frequency, and more quantitative CT emphysema than blood eosinophils [25]. As in our current study, neither group found robust associations between sputum and blood biomarkers. Although systemic markers are commonly consider to result from excess inflammation in the lung milieu that spillover into the circulation, it is increasingly recognized that markers present in pulmonary and systemic compartments may result from separate mechanisms [26]. Hence, our unexpected lack of relationships between plasma AcPGP and COPD outcomes in the current study may indicate that circulating AcPGP reflect processes unrelated to COPD. In addition to the neutrophil-chemoattractant properties of AcPGP, there is increasing evidence that AcPGP plays critical roles in endothelial dysfunction, angiogenesis, and cardiovascular injury [27, 28]. Thus, circulating AcPGP may reflect a cardiac or pulmonary vascular disease, conditions that are highly prevalent in COPD and should be studied in the future. Nevertheless, this work provides additional support for continuing pursuit of sputum-based biomarker panels for COPD.

Our study has limitations that deserve mention. First, participants with very severe COPD were excluded from sputum induction given concerns of safety in advanced disease. Thus, our findings reflect a moderate-to-severe COPD population, which remains highly relevant given that this group accounts for most individuals with COPD. Another limitation is the lack of a validation cohort. While this diminishes the generalizability of the findings, we and others have demonstrated the biological relevance of this matrikine pathway in the pathogenesis of COPD. Additionally, we only analyzed sputum AcPGP at one time point. However, we have previously shown that sputum AcPGP values remain consistent when measured repeatedly over a 12 week period [8]. Further, the small number of severe exacerbations increases the risk for type 1 error in our observations of increased odds of severe AECOPD and elevated sputum AcPGP. These findings should be validated in other cohorts. Finally, because of the observational nature of the study, we cannot determine the causality of the associations between elevated sputum AcPGP and features of COPD. Nevertheless, the known properties of AcPGP suggest that it warrants investigation as a potential therapeutic target to modify COPD progression.

\section{Conclusions}

Our data support sputum but not systemic AcPGP as associated with the severity of airflow limitation, emphysema and small airways disease, and risk for severe exacerbations in established COPD. Future prospective studies are needed to better elucidate the impact of elevated pulmonary AcPGP in patients at high risk for disease progression or exacerbations.

\section{Supplementary information}

Supplementary information accompanies this paper at https://doi.org/10. 1186/s12931-019-1230-8.

Additional file 1. Supplementary Methods. Figure S1. CONSORT

Diagram.

\section{Abbreviations}

ACPGP: Acetylated proline-glycine-proline; AECOPD: Acute exacerbation of COPD; CAT: COPD Assessment test; CB-SGRQ: Chronic Bronchitis defined by the SGRQ; FEV1: Forced expiratory volume in 1-s; FVC: Forced vital capacity; GOLD: Global initiative for chronic obstructive lung disease; MMRC: Modified medical research council; PRM-emph: Percent emphysema measured by parametric response mapping; PRM-fSAD: The percent functional small airways disease measured by parametric response mapping; SGRQ: St. George's Respiratory Questionnaire

\section{Acknowledgements}

SPIROMICS INVESTIGATORS:

\section{Authors' contributions}

JEB had full access to all of the data in the study and takes responsibility for the integrity of the data and accuracy of the analysis. JMW, TS, and JEB contributed to the conception and design of the study. JMW, DX, LV, YW, MTD, DC, WO'N , and JEB contributed to the acquisition of the data. JMW, DX, SPB, MTD, TS, and JEB contributed to the drafting of the manuscript. All authors contributed to revisions of the manuscript for critically important intellectual content and approved this version of the manuscript to be published.

\section{Funding}

K08 HL123940 and a research contract with Bayer AG to JMW; K24 HL140108 to MTD; R01 (R01HL102371, R01HL126596) and VA Merit (I01BX001756) to AG; R01 (R01HL114439, R01HL126596) and R35 (R35HL135710) to JEB; VA Merits 101 CX000911 to JLC and 101 CX001553 to CMF. SPIROMICS

(Subpopulations and Intermediate Outcomes in COPD Study) is funded by contracts from the NHLBI (HHSN268200900013C, HHSN268200900014C, HHSN268200900015C, HHSN268200900016C, HHSN268200900017C, HHSN268200900018C, HHSN268200900019C, HHSN268200900020C) and a grant from the $\mathrm{NIH} / \mathrm{NHLBI}(\mathrm{U} 01 \mathrm{HL} 137880)$, and supplemented by contributions made through the Foundation for the $\mathrm{NIH}$ and the COPD Foundation from AstraZeneca/Medlmmune; Bayer; Bellerophon Therapeutics; Boehringer-Ingelheim Pharmaceuticals, Inc.; Chiesi Farmaceutici S.p.A.; Forest Research Institute, Inc.; GlaxoSmithKline; Grifols Therapeutics, Inc.; Ikaria, Inc.; Novartis Pharmaceuticals Corporation; Nycomed GmbH; ProterixBio; Regeneron Pharmaceuticals, Inc.; Sanofi; Sunovion; Takeda Pharmaceutical Company; and Theravance Biopharma and Mylan.

\section{Availability of data and materials}

Interested investigators may request access to available data through processes outlined on the SPIROMICS website (https://www.spiromics.org/ spiromics/) under Obtaining SPIROMICS Data.

Ethics approval and consent to participate

This study was approved by the University of Alabama at Birmingham IRB (X110921005)

\section{Competing interests}

JMW has received grant support and consulting fees from the NIH, GSK, AZ, Gilead, Bayer, Quintiles, Mylan, Mereo BioPharma.

DX has no conflicts of interest.

LV has no conflicts of interest. 
RMB has no conflicts of interest.

YW has no conflicts of interest.

SPB has received grants from the $\mathrm{NIH}$.

MTD has received grants from the $\mathrm{NIH}$, American Lung Association, and the Department of Defense, Consulting fees from AstraZeneca, BI, GSK, Mereo, PneummRx/BTG and Quark, and contracted clinical trial support from AstraZeneca, Bl, Boston Scientific, GSK, Novartis PneumRx/BTG, Pulmonx, and Yungjin.

DC has no conflicts of interest.

WO'N has no conflicts of interest.

EAH has no conflicts of interest.

AG has received grant support from the $\mathrm{NIH}$ and Veterans Administration and consulting fees from Gilead Sciences, Grifols Inc., and Celtaxsys Inc. IB has no conflicts of interest.

JLC has received grants from NIH/NHLBI, NIH/NIAID, the Department of Veterans Affairs and the Department of Defense.

WWL has no conflicts of interest.

MKH has no conflicts of interest.

CMF has no conflicts of interest.

NP has no conflicts of interest.

TS is employed by Bayer AG and shareholder of Bayer AG.

JEB has no conflicts of interest.

\section{Author details}

'Division of Pulmonary, Allergy, and Critical Care Medicine, University of Alabama at Birmingham, Birmingham, AL, USA. ${ }^{2}$ UAB Lung Health Center, Birmingham, AL, USA. ${ }^{3}$ Birmingham VA Medical Center, Birmingham, AL, USA. ${ }^{4}$ Division of Pulmonary Diseases and Critical Care Medicine, University of North Carolina at Chapel Hill, Chapel Hill, NC, USA. ${ }^{5}$ Department of Medicine, University of North Carolina at Chapel HillMarsico Lung Institute/Cystic Fibrosis Research Center, Chapel Hill, NC, USA. ${ }^{6}$ Gillings School of Public Health, University of North Carolina at Chapel Hill, Chapel Hill, NC, USA. ${ }^{7}$ Department of Radiology, University of lowa Carver College of Medicine, lowa City, IA, USA. ${ }^{8}$ Division of Pulmonary and Critical Care Medicine, University of California-Los Angeles David Geffen School of Medicine, Los Angeles, CA, USA. ${ }^{9}$ Division of Pulmonary and Critical Care Medicine, University of Michigan-Ann Arbor, Ann Arbor, MI, USA. ${ }^{10}$ VA Ann Arbor Healthcare System, Ann Arbor, MI, USA. "'Division of Pulmonary and Critical Care Medicine, Johns Hopkins University, Baltimore, MD, USA. ${ }^{12}$ Bayer AG, AG, Pharmaceutical Division, Wuppertal, Germany.

Received: 13 June 2019 Accepted: 1 November 2019

Published online: 12 November 2019

\section{References}

1. Vogelmeier CF, Criner GJ, Martinez FJ, Anzueto A, Barnes PJ, Bourbeau J, Celli BR, Chen R, Decramer M, Fabbri LM, et al. Global Strategy for the Diagnosis, Management, and Prevention of Chronic Obstructive Lung Disease 2017 Report. GOLD executive summary. Am J Respir Crit Care Med. 2017(195):557-82.

2. Mannino DM. Biomarkers for chronic obstructive pulmonary disease diagnosis and progression: insights, disappointments and promise. Curr Opin Pulm Med. 2019;25:144-9.

3. Weathington $N M$, van Houwelingen $A H$, Noerager $B D$, Jackson $P L$, Kraneveld AD, Galin FS, Folkerts G, Nijkamp FP, Blalock JE. A novel peptide CXCR ligand derived from extracellular matrix degradation during airway inflammation. Nat Med. 2006;12:317-23.

4. Wells JM, O'Reilly PJ, Szul T, Sullivan DI, Handley G, Garrett C, McNicholas CM, Roda MA, Miller BE, Tal-Singer $\mathrm{R}$, et al. An aberrant leukotriene A4 hydrolase-proline-glycine-proline pathway in the pathogenesis of chronic obstructive pulmonary disease. Am J Respir Crit Care Med. 2014;190:51-61.

5. Gaggar A, Jackson PL, Noerager BD, O'Reilly PJ, McQuaid DB, Rowe SM, Clancy JP, Blalock JE. A novel proteolytic cascade generates an extracellular matrix-derived chemoattractant in chronic neutrophilic inflammation. J Immunol. 2008;180:5662-9.

6. O'Reilly PJ, Hardison MT, Jackson PL, Xu X, Snelgrove RJ, Gaggar A, Galin FS, Blalock JE. Neutrophils contain prolyl endopeptidase and generate the chemotactic peptide, PGP, from collagen. J Neuroimmunol. 2009;217:51-4.

7. Snelgrove RJ, Jackson PL, Hardison MT, Noerager BD, Kinloch A, Gaggar A, Shastry S, Rowe SM, Shim YM, Hussell T, Blalock JE. A critical role for
LTA4H in limiting chronic pulmonary neutrophilic inflammation. Science. 2010;330:90-4.

8. Wells JM, Jackson PL, Viera L, Bhatt SP, Gautney J, Handley G, King RW, Xu X, Gaggar A, Bailey WC, et al. A Randomized, Placebo-controlled Trial of Roflumilast Effect on Proline-glycine-Proline and Neutrophilic inflammation in chronic obstructive pulmonary disease. Am J Respir Crit Care Med. 2015;192:934-42.

9. Couper D, LaVange LM, Han M, Barr RG, Bleecker E, Hoffman EA, Kanner R, Kleerup E, Martinez FJ, Woodruff PG, et al. Design of the Subpopulations and Intermediate Outcomes in COPD study (SPIROMICS). Thorax. 2014:69:491-4.

10. Celli BR, MacNee W, Force AET. Standards for the diagnosis and treatment of patients with COPD: a summary of the ATS/ERS position paper. Eur Respir J. 2004:23:932-46.

11. Freeman CM, Crudgington S, Stolberg VR, Brown JP, Sonstein J, Alexis NE, Doerschuk CM, Basta PV, Carretta EE, Couper DJ, et al. Design of a multicenter immunophenotyping analysis of peripheral blood, sputum and bronchoalveolar lavage fluid in the subpopulations and intermediate outcome measures in COPD study (SPIROMICS). J Transl Med. 2015;13:19.

12. O'Reilly PJ, Jackson PL, Wells JM, Dransfield MT, Scanlon PD, Blalock JE, Sputum PGP is reduced by azithromycin treatment in patients with COPD and correlates with exacerbations. BMJ Open. 2013;3:e004140.

13. Jones PW, Harding G, Berry P, Wiklund I, Chen WH, Kline Leidy N. Development and first validation of the COPD assessment test. Eur Respir J. 2009;34:648-54

14. Jones PW, Quirk FH, Baveystock CM: The St George's Respiratory Questionnaire. Respir Med 1991, 85 Suppl B:25-31; discussion 33-27.

15. Cotes JE. Medical Research Council Questionnaire on Respiratory Symptoms (1986). Lancet. 1987(2):1028.

16. Kim V, Crapo J, Zhao H, Jones PW, Silverman EK, Comellas A, Make BJ, Criner GJ, Investigators CO. Comparison between an alternative and the classic definition of chronic bronchitis in COPDGene. Ann Am Thorac Soc. 2015;12:332-9.

17. Laboratories ATSCOPSFCPF. ATS statement: guidelines for the six-minute walk test. Am J Respir Crit Care Med. 2002;166:111-7.

18. McGhan R, Radcliff T, Fish R, Sutherland ER, Welsh C, Make B. Predictors of rehospitalization and death after a severe exacerbation of COPD. Chest. 2007;132:1748-55.

19. Han MK, Quibrera PM, Carretta EE, Barr RG, Bleecker ER, Bowler RP, Cooper $C B$, Comellas A, Couper DJ, Curtis JL, et al. Frequency of exacerbations in patients with chronic obstructive pulmonary disease: an analysis of the SPIROMICS cohort. Lancet Respir Med. 2017;5:619-26.

20. Sieren JP, Newell JD Jr, Barr RG, Bleecker ER, Burnette N, Carretta EE, Couper D, Goldin J, Guo J, Han MK, et al. SPIROMICS protocol for multicenter quantitative computed tomography to phenotype the lungs. Am J Respir Crit Care Med. 2016;194:794-806.

21. Boes JL, Hoff BA, Bule M, Johnson TD, Rehemtulla A, Chamberlain R, Hoffman EA, Kazerooni EA, Martinez FJ, Han MK, et al. Parametric response mapping monitors temporal changes on lung $C T$ scans in the subpopulations and intermediate outcome measures in COPD study (SPIROMICS). Acad Radiol. 2015;22:186-94.

22. Galban CJ, Chenevert TL, Meyer CR, Tsien C, Lawrence TS, Hamstra DA, Junck L, Sundgren PC, Johnson TD, Ross DJ, et al. The parametric response map is an imaging biomarker for early cancer treatment outcome. Nat Med. 2009;15:572-6.

23. Vasilescu DM, Martinez FJ, Marchetti N, Galban CJ, Hatt C, Meldrum CA, Dass C, Tanabe N, Reddy RM, Lagstein A, et al. Non-invasive imaging biomarker identifies small airway damage in severe COPD. Am J Respir Crit Care Med. 2019.

24. Singh D, Edwards L, Tal-Singer R, Rennard S. Sputum neutrophils as a biomarker in COPD: findings from the ECLIPSE study. Respir Res. 2010;11:77.

25. Hastie AT, Martinez FJ, Curtis JL, Doerschuk CM, Hansel NN, Christenson S, Putcha N, Ortega VE, Li X, Barr RG, et al. Association of sputum and blood eosinophil concentrations with clinical measures of COPD severity: an analysis of the SPIROMICS cohort. Lancet Respir Med. 2017;5:956-67.

26. Wouters EF, Reynaert NL, Dentener MA, Vernooy JH. Systemic and local inflammation in asthma and chronic obstructive pulmonary disease: is there a connection? Proc Am Thorac Soc. 2009:6:638-47.

27. Hill JW, Nemoto EM. Matrix-derived inflammatory mediator N-acetyl prolineglycine-proline is neurotoxic and upregulated in brain after ischemic stroke. J Neuroinflammation. 2015;12:214. 
28. Payne GA, Li J, Xu X, Jackson P, Qin H, Pollock DM, Wells JM, Oparil S, Leesar M, Patel RP, et al. The Matrikine acetylated Proline-glycine-Proline couples vascular inflammation and acute cardiac rejection. Sci Rep. 2017;7:7563.

\section{Publisher's Note}

Springer Nature remains neutral with regard to jurisdictional claims in published maps and institutional affiliations.

Ready to submit your research? Choose BMC and benefit from:

- fast, convenient online submission

- thorough peer review by experienced researchers in your field

- rapid publication on acceptance

- support for research data, including large and complex data types

- gold Open Access which fosters wider collaboration and increased citations

- maximum visibility for your research: over $100 \mathrm{M}$ website views per year

At $B M C$, research is always in progress. 